\title{
Efecto de la fertilización fosfatada en la fenología de tres variedades de alfalfas (Medicago sativa L.) @(1) (8)
}

\author{
Effect of phosphatic fertilization on the phenology of three varieties of alfalfa \\ (Medicago sativa L.)
}

Wilson Oñate Viteri. ${ }^{1}$ \& Enrique Flores Mariazza. ${ }^{2}$

Recibido: 14-04-2019 / Revisado: 20-05-2019 /Aceptado: 18-06-2019/ Publicado: 05-07-2019

\begin{abstract}
.
DOI: https://doi.org/10.33262/cienciadigital.v3i3.622

The effect of different levels of phosphate fertilization on three varieties of alfalfa introduced in three phenological stages in the agronomic behavior was determined. The trial was carried out at the Tunshi Experimental Station, located in the canton of Riobamba, Province of Chimborazo, Ecuador. The treatments that were used were the varieties of alfalfa as factor A (abundant green, cuf-101 and sw-8210) and as factor B the fertilization doses $(0,50,100$ and $150 \mathrm{~kg} / \mathrm{P} / \mathrm{ha})$. The experimental plots were distributed under a completely randomized block design according to split plots and three repetitions; analysis of variance was performed, comparison of means according to Tukey and regression analysis. The experimental results did not register significant differences $(p>0.05)$ for the ineraction $\mathrm{A} \times \mathrm{B}$. When analyzing the main factors in factor $\mathrm{A}$, it was observed that the leaf / stem ratio for the green abundant variety was 1.23 leaves / stem, differing significantly $(\mathrm{p}<0.05)$ from the variety sw8210 that registered 1.12 leaves / stem. When analyzing factor B, air coverage reached $84.29 \%$ when $150 \mathrm{~kg}$ / $\mathrm{P} /$ ha was applied, which differed significantly $(\mathrm{p}<0.01)$ from control treatment (76.60\%). In addition, the use of $150 \mathrm{~kg} / \mathrm{P} / \mathrm{ha}$ recorded a baseline coverage of $29.41 \%$, which differed statistically $(\mathrm{p}<0.01)$ from the control treatment $(25.74 \%)$. The leaves / stem ratio was 1.26 significantly different $(\mathrm{p}<0.01)$ from the control treatment $(1.11)$. Regarding dry matter production, no significant differences were found between alfalfa varieties and levels of phosphate fertilization $(\mathrm{P}>0.05)$, making the harvest time at 110 days when the plants presented more than five sprouts since there were no induction to flowering. The economic analysis determined a benefit / cost of $\$ 1.38$ when $150 \mathrm{~kg} / \mathrm{P}$ / ha was used, a value that differed from the control treatment $(\mathrm{P}<0.01)$ with a benefit / cost of $\$ 1.23$.
\end{abstract}

\footnotetext{
${ }^{1}$ Escuela Superior Politécnica de Chimborazo, Riobamba, Ecuador. wilsonovec@yahoo.es

${ }^{2}$ Universidad Nacional Agraria, Laboratorio de Ecología y Utilización de Pastizales, La Molina, Lima, Perú, wilsonovec@yahoo.es
} 
Keywords: Economic Analysis, Alfalfa Varieties, Phosphate Fertilization

\section{Resumen}

Se determinó el efecto de diferentes niveles de fertilización fosfatada en tres variedades de alfalfas introducidas en tres etapas fenológicas en el comportamiento agronómico. El ensayo se realizó en la Estación Experimental Tunshi, ubicada en el cantón Riobamba, Provincia de Chimborazo, Ecuador. Los tratamientos que se utilizaron fueron las variedades de alfalfa como factor A (abunda verde, cuf-101 y sw-8210) y como factor B las dosis de fertilización $(0,50,100$ y $150 \mathrm{~kg} / \mathrm{P} / \mathrm{ha})$. Las parcelas experimentales se distribuyeron bajo un diseño de bloques completamente al azar con arreglo en parcelas divididas y tres repeticiones; se realizó el análisis de varianza, comparación de medias según Tukey y el análisis de la regresión. Los resultados experimentales no registraron diferencias significativas $(\mathrm{p}>0.05)$ para la interacción A x . Al analizar los factores principales en el factor A se observó que la relación hojas/tallo para la variedad abunda verde fue de 1.23 hojas/tallo, difiriendo significativamente $(\mathrm{p}<0.05)$ de la variedad sw8210 que registró 1.12 hojas/tallo. Al analizar el factor $\mathrm{B}$, la cobertura aérea alcanzó el $84.29 \%$ cuando se aplicó $150 \mathrm{~kg} / \mathrm{P} / \mathrm{h}$ a que difirió significativamente $(\mathrm{p}<0.01)$ del tratamiento control (76.60\%). Además la utilización de $150 \mathrm{~kg} / \mathrm{P} / \mathrm{ha}$ registró una cobertura basal de $29.41 \%$ que difirió estadísticamente $(\mathrm{p}<0.01)$ del tratamiento control (25.74\%). La relación hojas/tallo fue de 1.26 diferenciándose significativamente $(\mathrm{p}<0.01)$ del tratamiento testigo (1.11). En relación a la producción de materia seca no se evidencio diferencias significativas entre variedades de alfalfa y los niveles de fertilización fosfatada $(\mathrm{P}>0.05)$, realizando la época de cosecha a los 110 días cuando las plantas presentaron más de cinco rebrotes ya que no hubo inducción a la floración. El análisis económico determinó un benéfico/costo de $\$ 1.38$ cuando se utilizó 150 $\mathrm{kg} / \mathrm{P} / \mathrm{ha}$ valor que difirió del tratamiento control $(\mathrm{P}<0.01)$ con un beneficio/costo de $\$$ 1.23 .

Palabras clave: Análisis Económico, Variedades De Alfalfa, Fertilización Fosfatada

\section{Introducción}

La alfalfa (Medicago sativa L.) es una leguminosa forrajera perenne cultivada en todas las regiones del mundo en climas subtropical, templado y seco (Liu et al., 2015); la importancia de la alfalfa se debe a su potencial de producción y valor nutritivo, y a su utilización como forraje verde, heno, ensilado, pellets y otros (Milic et al., 2014; Rojas et al., 2017); por lo que se han realizado muchos esfuerzos en investigaciones para su mejora genética (Marijana et al. 2011). La alfalfa tiene una alta calidad nutricional y producción de biomasa (Rogers et al., 2014), se adapta ampliamente a diversos climas ( Avci et al., 2013 ) y es más efectiva que los cultivos anuales para reducir la escorrentía y la erosión del suelo (Fan et al., 2014). Sin embargo el rendimiento y los contenidos de nutrientes de la alfalfa pueden verse afectados principalmente en suelos salinos (Ensiye et al., 2018). En la actualidad los programas de mejoramiento de alfalfa se han centrado en el rendimiento y la calidad del forraje, la resistencia 
a los factores estresantes bióticos y abióticos y la falta de agua (Hawkins, 2018). El agua es un factor limitante para la producción de cultivos en muchas partes del mundo (Raza, 2014).

En la última encuesta de superficie y producción agropecuaria realizada por el Instituto Nacional de Estadística y Censos (INEC, 2009) reporta que en la provincia de Chimborazo de las 523.340 ha de uso de suelo, 79.951 ha $(15,22 \%)$ se utilizan para pastos cultivados y se ellas 5250 hectáreas se destinan al cultivo de la alfalfa, lo que constituye el 6, 57\%. Grijalva (1995), manifiesta que el cultivo de alfalfa en el Ecuador en las explotaciones medianas y pequeñas se lo viene realizando de manera relativa empírica, en donde el uso de fertilizantes, variedades mejoradas, riegos y manejos adecuados de cortes no son tareas cotidianas, lo que han conducido a obtener bajos rendimientos productivos. En el Ecuador, lamentablemente los ganaderos se dedican únicamente a la producción de forraje verde utilizando semillas importadas de otros países ya que es conocido que la mayoría de las variedades que se ofrecen provienen principalmente de Estados Unidos y Nueva Zelanda (Alaska, 2015) debido a que el país no dispone de un programa oficial de semillas de pastos y forrajes que provea una continua producción y un suministro oportuno de semilla de calidad y de variedades adaptadas a los diferentes tipos de suelos para mejorar la productividad de estos cultivos. Entre los años 2000 y 2013 el Ecuador importó más de 1.700 toneladas métricas de semillas de alfalfa, de las cuales el 93\% provinieron de Estados Unidos (Banco Central del Ecuador, 2014).

Sanz et al. (2017), indicaron que el nitrógeno, junto con el fósforo son los macronutrientes principales de las plantas que limitan el crecimiento de la alfalfa. Aunque el $\mathrm{P}$ es abundante en muchos suelos, su disponibilidad para las plantas es baja. El cultivo de alfalfa requiere de suelos con $\mathrm{pH}$ neutro, textura media a liviana, buen drenaje y profundidad, con alta disponibilidad de fósforo, donde puede expresar todo su potencial productivo. En este sentido la deficiencia de fosfato es un factor nutricional importante que limita la producción de las leguminosas, particularmente en suelos ácidos y calcáreos (Martins et al., 2017). Por ello, el análisis químico de la capa arable es imprescindible para conocer la situación particular y orientar los programas de fertilización. Los beneficios de la fertilización fosfatada inicial de la alfalfa se perciben, entre otros, en la mayor formación de nódulos, en el estímulo del crecimiento inicial, mayor desarrollo de las raíces, mejor competencia con las arvenses y en el anticipo del primer aprovechamiento.

Por otra parte la fenología de la alfalfa estudia y describe los diferentes eventos fenológicos que se dan en las especies vegetales dentro de ecosistemas naturales o agrícolas en su interacción con el medio ambiente. Numerosos trabajos han demostrado que a medida que la alfalfa avanza en su estado de madurez su calidad disminuye (Soto y Jahn, 1993; Ruiz et al., 1994). Esta variación en el estado de madurez, de acuerdo al estado fenológico de la planta, determina el aporte de nutrientes que satisfacen los distintos requerimientos de vacas de diferente potencial productivo (Jagush et al., 1976), con estos antecedentes el objetivo de la presente investigación 
es determinar el efecto de la fertilización fosfatada en diferentes variedades de alfalfas introducidas en tres etapas fenológicas en el comportamiento agronómico.

\section{Materiales y Métodos}

El presente estudio se realizó en la Estación Experimental Tunshi, perteneciente a la Facultad de Ciencias Pecuarias, de la Escuela Superior Politécnica de Chimborazo (ESPOCH), ubicada en el kilómetro 12 vía a Licto, Provincia de Chimborazo, con una longitud de $79^{0} 40^{\prime}$ oeste y latitud $01^{0} 65^{\prime}$ sur, y a una altitud de $2754 \mathrm{msnm}$, la temperatura está entre 10 a $22^{\circ} \mathrm{C}$ con una media de $13.5^{\circ} \mathrm{C}$, la precipitación anual varía entre 550 a $800 \mathrm{~mm}$, con una humedad relativa entre 48 a 96\% con una media del 80\% (INAMHI, 2017). El ensayo se realizó durante la campaña agrícola diciembre 2016 a marzo 2017 (tabla1).

Tabla 1. Temperatura, precipitación y humedad relativa de la Estación Experimental Tunshi.

\begin{tabular}{lcccc}
\hline Variable & Diciembre 2016 & Enero 2017 & Febrero 2017 & Marzo 2017 \\
\hline Temperatura ${ }^{\circ} \mathrm{C}$ & 13.90 & 13.40 & 13.40 & 13.10 \\
Precipitación mm & 49.70 & 88.10 & 1240 & 150.20 \\
Humedad Relativa & 75.80 & 79.30 & 79.50 & 84.60 \\
$\%$ & & & & \\
\hline
\end{tabular}

\section{Caracterización del suelo}

En la tabla 2, se indica el análisis de caracterización de suelos de la Estación Experimental Tunshi. El suelo de este sitio posee un $\mathrm{pH}$ neutro $(7,4)$ y con un bajo nivel de $\mathrm{P}(7,5 \mathrm{ppm})$ en la capa arable, la topografía es plana (1,5\% de pendiente) y la estructura del suelo es franco arenoso con un buen drenaje.

Tabla 2. Caracterización de suelos de la Estación Experimental Tunshi

\begin{tabular}{cccccc}
\hline Parámetros & $\mathrm{pH}$ & $\mathrm{MO}(\%)$ & $\mathrm{N}(\%)$ & $\mathrm{P}(\mathrm{ppm})$ & $\mathrm{K}(\mathrm{c} \mathrm{mol} / \mathrm{kg})$ \\
\hline & $7.4 \pm 0.010$ & $2.53 \pm 0.020$ & $0.33 \pm 0.013$ & $7.8 \pm 0,011$ & $0.86 \pm 0.012$ \\
\cline { 2 - 6 } & $\mathrm{Ca}(\mathrm{c} \mathrm{mol} / \mathrm{kg})$ & $\mathrm{Mg}(\mathrm{c} \mathrm{mol} / \mathrm{kg})$ & $\mathrm{Fe}(\mathrm{ppm})$ & $\mathrm{Mn}(\mathrm{ppm})$ & $\mathrm{Cu}(\mathrm{ppm})$ \\
\cline { 2 - 6 } & $9.52 \pm 0.008$ & $3.55 \pm 0,007$ & $65.1 \pm 0.005$ & $7.16 \pm 0.001$ & 9.610 .004 \\
\hline
\end{tabular}

Fuente: Laboratorio de Análisis de suelos y fertilizantes de Agrocalidad (2016).

\section{Diseño Experimental}

Los tratamientos que se utilizaron fueron las variedades de alfalfa, como factor A (abunda verde, cuf-101 y sw8210) y como factor B las dosis de fertilización $(0,50,100$ y $150 \mathrm{~kg} / \mathrm{ha})$ con tres 
repeticiones; los cuales se analizaron bajo un diseño de bloques completamente al azar con arreglo en parcelas divididas que se ajustó al siguiente modelo lineal aditivo:

$Y i j k=\mu+R i+A j+A i R j+B k+A j B k+E i j k$

\section{dónde:}

Yijk: Valor estimado de la variable

$\mu$ : $\quad$ Media general

Ri: $\quad$ Efecto de los bloques (R)

Aj: $\quad$ Efecto de las variedades de alfalfas (A)

$\mathrm{AiRj}$ : Efecto de la interacción entre las repeticiones y las variedades (AR)

Bk: Efecto de los niveles de fertilización fosfatada (B)

AjBk: Efecto de la interacción variedades de alfalfa y fertilización fosfatada (AB)

Eijk: Efecto residual.

Una vez seleccionado el sitio se comenzó con la preparación del suelo donde se estableció el cultivo de alfalfa, el mismo que consistió en 3 pasos: arado, rastrado y nivelación del terreno, luego se limitaron las parcelas con áreas de 5 x $4 \mathrm{~m}\left(20 \mathrm{~m}^{2}\right)$ que fue la unidad experimental, con una separación entre bloques de $1 \mathrm{~m}^{2}$ de distancia, las mismas que se delimitaron con estacas, para el estudio se contó con tres bloques conformadas por 12 parcelas cada una, sumado un área total de $720 \mathrm{~m}^{2}$ con 36 parcelas. Se partió con una muestra de suelo para realizar el análisis químico y físico del suelo antes de la siembra.

La siembra se realizó al voleo con semilla certificada de alfalfa de tres variedades de origen de los Estados Unidos (abunda verde, cuf101 y sw8210), con una densidad de siembra de 50 lb/ha; la fertilización química $(0,50,100$ y $150 \mathrm{~kg} / \mathrm{ha})$ se realizó al momento de la siembra utilizando el superfosfato triple (SFT) como fuente de fósforo, la siembra se realizó en el mes de diciembre de 2016. Antes de la siembra cada variedad de alfalfa se sorteó aleatoriamente en cada bloque (parcela principal) y en cada variedad de alfalfa de cada bloque (subparcela) se sorteó aleatoriamente los niveles de fósforo.

Las labores culturales fueron homogéneas para todas las unidades experiméntales principalmente el control de malezas y el riego de agua fue acorde a las condiciones ambientales imperantes en la Estación Experimental Tunshi. Las evaluaciones se realizaron en tres etapas fenológicas: primera etapa de emergencia, segunda de desarrollo y la tercera de cosecha, teniendo las siguientes variables: Longitud de raíz, altura de la planta, porcentaje de cobertura aérea y basal, número de tallos por planta, número de plantas $\mathrm{m}^{2}$, número y altura de rebrotes, porcentaje composición botánica, relación tallo/hojas, producción de forraje verde y materia seca (t/corte), días a la cosecha y el beneficio/costo. 


\section{Marco metodológico}

Análisis de suelo. Las muestras obtenidas de suelo se analizaron en el Laboratorio de Agrocalidad del Ministerio de Agricultura (Agrocalidad, 2016).

Porcentaje de germinación. Se evaluó el porcentaje de plantas que han prendido y/o germinado a los 21 días después de la siembra (Gillén, et. al. 2010).

Longitud de la raíz. Se midió con una regla graduada a los 21 días, es decir cuando se calculó el porcentaje de germinación.

Altura de planta $(\mathrm{cm})$. La altura de la planta se tomó como la distancia media comprendida entre la parte basal del tallo hasta el ápice de la hoja (altura de la canopía).

Cobertura basal (\%). La cobertura basal se evaluó con el método de la transecta permanente de Parker (Parker 1954).

Número de tallos por planta. Se determinó con el conteo manual de cada uno de los tallos de 10 plantas al azar, este parámetro se realizó por cada tratamiento y se calculó los promedios (Toledo 1982).

Número plantas $\mathrm{m}^{2}$. Se utilizó un cuadrante de $0,5 \mathrm{~m}^{2}$, para lo cual se realizó el conteo manual del número de plantas en cada cuadrante y luego se expresó en plantas por $\mathrm{m}^{2}$.

Número y altura de nuevos rebrotes. Cuando por razones climáticas no hay inducción de la floración (Harris, 1978) indica que la utilización del cultivo es en base al conteo manual de los nuevos rebrotes de la corona y cuando alcancen entre 5-7 $\mathrm{cm}$ de altura.

Composición botánica. Se calculó la composición florística sobre la base de cobertura vegetal en porcentaje $(\%)$.

Relación hojas/tallos. Se utilizaron submuestras de las muestras obtenidas para rendimiento de forraje. Se separaron en los componentes morfológicos (hojas y tallos), se secaron y se pesaran para posteriormente estimar el porcentaje de hoja y tallo en la muestra. La relación hoja/tallo se determinó al dividir el rendimiento de hoja entre el de tallo cuándo la planta emitió 4 a 5 brotes nuevos (León, 2003) ya que no hubo inducción de la floración.

Producción de materia seca (t/ha/corte). La producción de cada variedad de forraje verde se expresó en ( $\mathrm{t} / \mathrm{ha} /$ corte), para ello se tomó el peso de la muestra verde en un metro cuadrado; y este valor se llevó a su equivalencia en producción de materia seca por hectárea (Hoyos et al.1996). 
Frecuencia de corte. Se determinó individualmente para cada cultivar tomando en cuenta el cuándo la planta emitió 4 a 5 brotes nuevos (León 2003) ya que no hubo inducción de la floración. La alfalfa se debe cortar entre los 5 a $7 \mathrm{~cm}$ sobre la superficie del suelo, ya que a esa altura no se daña la corona de la planta ni los rebrotes, los cuales serán el forraje del siguiente corte (Infoagro, 2005).

Análisis Beneficio/costo. Se realizó comparando entre los ingresos totales que está en base a la producción de forraje verde de la alfalfa de cada uno de los tratamientos sobre los egresos totales del cultivo.

\section{Análisis estadístico}

Los resultados obtenidos en la investigación fueron tabulados en una hoja electrónica Excel de Office 2016, posteriormente se realizó el procesamiento estadístico para lo cual se utilizó el paquete estadístico (Infostat, 2017). Los resultados obtenidos fueron sometidos a los siguientes análisis: Análisis de Variancia (ADEVA), Separación de medias según Tukey $\mathrm{P}<0.05$ y Análisis de regresión y correlación.

\section{Análisis de Resultados y Discusión}

Los resultados experimentales no registraron diferencias significativas $(\mathrm{P}>0.05)$ para la interacción (A x B) variedades de alfalfa introducidas abunda verde, cuf-101 y Sw-8210 con los niveles de fertilización fosfatada $0,50,100$ y $150 \mathrm{~kg} / \mathrm{P} / \mathrm{ha}$, por lo que se analizó los efectos de los factores principales.

\section{Comportamiento agronómico y fenológico de las variedades de alfalfa (factor a) Etapa de emergencia}

En la tabla 2 se indica la etapa de emergencia donde se observa que el porcentaje de germinación, la longitud de la raíz y los días a la germinación no presentaron diferencias estadísticas significativas ( $>$ > 0.05) para las variedades de alfalfa abunda verde, cuf101 y sw8210. Lo que indica que el comportamiento agronómico fue similar en esta etapa.

Tabla 1. Respuesta agronómica de tres variedades de Medicago sativa (alfalfa) en la etapa de emergencia

\begin{tabular}{lcccccccc}
\hline & \multicolumn{9}{c}{ Variedades } & & \\
\cline { 2 - 7 } \multicolumn{1}{c}{ Variables } & Abunda Verde & & Cuf101 & & Sw8210 & E.E. & Prob. \\
\hline Germinación (\%) & 93.75 & a & 95.17 & a & 94.50 & a & 0.34 & 0.10 \\
Longitud raíz 30 días (cm.) & 5.25 & a & 5.25 & a & 4.92 & a & 0.35 & 0.75 \\
Días germinación & 19.75 & a & 20.17 & a & 19.92 & a & 0.23 & 0.49 \\
\hline
\end{tabular}

Letras iguales no difieren significativamente según Tukey $(\mathrm{P}<0.05)$. 


\section{Etapa de desarrollo}

En la tabla 3, se indica el número de días para la formación de los tres foliolos, días al primer macollo, altura macollos 40, 60 y 90 días de edad y número macollos 60 y 90 días de edad, en la cual se observa que no existen diferencias significativas $(p>0.05)$ para las variedades de alfalfa abunda verde, cuf101 y esto se debe a que las condiciones climáticas y edáficas fueron homogéneas, además el genotipo de las tres variedades de alfalfa respondieron de la misma manera.

Tabla 2. Respuesta agronómica de tres variedades de Medicago sativa (alfalfa) en la etapa de desarrollo.

\begin{tabular}{lccccccccc}
\hline & \multicolumn{9}{c}{ Variedades } \\
\cline { 2 - 7 } \multicolumn{1}{c}{ Variables } & Abunda & & & & & & \\
Derde & & Cuf101 & & Sw 8210 & & E.E. & Prob. \\
\hline Días tres foliolos & 24.58 & a & 25.08 & a & 25.50 & a & 0.20 & 0.08 \\
Aías primer macollo & 33.25 & a & 33.33 & a & 33.58 & a & 0.54 & 0.90 \\
Altura macollos 40 días (cm.) & 11.91 & a & 12.37 & a & 12.52 & a & 0.29 & 0.40 \\
Número macollos 60 días (cm.) & 3.42 & a & 3.17 & a & 3.17 & a & 0.43 & 0.90 \\
Altura macollos 60 días (cm.) & 23.79 & a & 23.43 & a & 23.46 & a & 0.45 & 0.83 \\
Número tallos 90 días (No.) & 8.08 & a & 7.44 & a & 8.22 & a & 0.27 & 0.21 \\
Altura planta 90 días (cm.) & 45.37 & a & 44.19 & a & 46.30 & a & 1.05 & 0.44 \\
\hline
\end{tabular}

Letras iguales no difieren significativamente según Tukey $(\mathrm{P}<0.05)$.

\section{Etapa cosecha (110 días)}

En la tabla 4 se reporta la altura de la planta, número de plantas por $\mathrm{m}^{2}$, número y altura de los rebrotes $(\mathrm{cm})$, cobertura aérea y basal (\%), composición botánica (\%), longitud de la raíz y beneficio/costo; variables que no presentan diferencias significativas ( $>$ >05) para las variedades de alfalfa abunda verde, cuf101.

Tabla 3. Respuesta agronómica de tres variedades de Medicago sativa (alfalfa) en la etapa de cosecha.

\begin{tabular}{lccccccccc}
\hline & \multicolumn{9}{c}{ Variedades } \\
\cline { 2 - 8 } \multicolumn{1}{c}{ Variables } & $\begin{array}{c}\text { Abunda } \\
\text { Verde }\end{array}$ & Cuf101 & & Sw8210 & & E.E. & Prob. \\
\hline Altura planta (cm.) & 53.59 & a & 53.06 & a & 55.42 & a & 0.89 & 0.26 \\
Número plantas m $^{2}$ & 20.92 & a & 18.00 & a & 18.58 & a & 0.94 & 0.18 \\
Número rebrotes (cm.) & 5.08 & a & 4.92 & a & 4.67 & a & 0.10 & 0.09 \\
Altura rebrotes (cm.) & 6.33 & a & 7.00 & a & 6.92 & a & 0.49 & 0.61 \\
Cobertura aérea (\%) & 80.44 & a & 77.19 & a & 83.19 & a & 1.93 & 0.21 \\
\hline
\end{tabular}


Vol. 3, N³, p. 178-194, julio - septiembre, 2019

\begin{tabular}{lcccccccc}
\hline Cobertura basal (\%) & 28.14 & $\mathrm{a}$ & 27.39 & $\mathrm{a}$ & 27.13 & $\mathrm{a}$ & 0.50 & 0.41 \\
Composición botánica (\%) & 93.89 & $\mathrm{a}$ & 94.83 & $\mathrm{a}$ & 94.50 & $\mathrm{a}$ & 0.54 & 0.52 \\
Relación hoja/tallo & 1.23 & $\mathrm{a}$ & 1.20 & $\mathrm{~b}$ & 1.12 & $\mathrm{c}$ & 0.02 & 0.03 \\
Producción forraje verde (t/ha.) & 8.44 & $\mathrm{a}$ & 9.97 & $\mathrm{a}$ & 9.91 & $\mathrm{a}$ & 0.83 & 0.42 \\
Producción materia seca (t/ha.) & 2.15 & $\mathrm{a}$ & 2.43 & $\mathrm{a}$ & 2.43 & $\mathrm{a}$ & 0.18 & 0.51 \\
Longitud raíz (cm.) & 18.50 & $\mathrm{a}$ & 18.58 & $\mathrm{a}$ & 17.17 & $\mathrm{a}$ & 1.09 & 0.62 \\
Beneficio / Costo (\$) & 1.21 & $\mathrm{a}$ & 1.54 & $\mathrm{~b}$ & 1.21 & $\mathrm{a}$ & 0.02 & 0.01 \\
\hline
\end{tabular}

Letras iguales no difieren significativamente según Tukey $(\mathrm{P}<0.05)$.

\section{Relación Hoja/Tallo}

La variedad abunda verde registró una relación hojas / tallo de 1.23, valor que difirió significativamente $(\mathrm{p}<0.05)$ de las variedades cuf101 y sw8210, con 1.20 y 1.12 hojas por tallo respectivamente. Esto posiblemente se deba a la genética de cada una de las variedades ya que las hojas de alfalfa son los componentes de la planta que presentan el mayor valor nutritivo y potencial de consumo en el momento del corte (10\% floración o $5 \mathrm{~cm}$. de rebrote basal), por lo tanto la calidad de esta especie forrajera puede ser mejorada con cultivares que presenten una mayor cantidad de hojas. Romero, (2002) en un experimento para evaluar la evolución de la producción de materia seca y la relación hojas/tallo como un estimador de la calidad de cultivares de alfalfa trifoliadas y multifoliadas con distinto grado de reposo, encontró una relación hojas/tallo, en los momentos de corte temprano, medio y tardío cuyos valores fueron $1.43,1.20$ y 0.60 respectivamente, las mismas que son valores extremos a los alcanzados en el presente estudio.

\section{Producción forraje verde y materia seca (t/ha.)}

La producción de forraje verde y materia seca en las variedades de alfalfa abunda verde, cuf101 y sw8210 se observó que no existen diferencias significativas ( $p>0.05)$ en las tres variedades de alfalfa, reportándose producciones entre 8.44 - 9.97 (t/ha/fv/corte) y de $2.15-2.43$ (t/ha/ms/corte) lo que indicaría que el manejo agronómico fue homogéneo y la expresión genética de las tres variedades fueron similares. La producción forrajera está definida en gran medida, por las características genéticas del cultivar, y aunque esto determine un rendimiento potencial elevado, es necesario que se den las condiciones necesarias para que éste pueda expresarse. En este aspecto, el ambiente y el manejo, juegan un rol de fundamental importancia en la definición del rendimiento logrado.

\section{Beneficio/costo $(\$)$}

El beneficio/costo de la variedad de alfalfa cuf101 fue de 1.54 dólares, valor que difirió significativamente $(\mathrm{p}<0.05)$ de las variedades abunda verde y sw8210 ya que estas especies registraron un beneficio/costo de 1.21 dólares; lo que quiere decir que por cada dólar invertido se obtendría una ganancia entre \$0.54 y/o \$ 0.21. La inversión en fertilizantes representa una 
Vol. 3, N³, p. 178-194, julio - septiembre, 2019

parte importante de los costos de producción de este cultivo forrajero; por esto, una fertilización lo más próxima a las necesidades reales de las plantas, contribuirá por una parte a que éstas no se vean restringidas en su crecimiento por limitaciones de nutrientes, y por otra, que la inversión en fertilizantes sea sólo la necesaria para obtener un retorno adecuado.

\section{Comportamiento de la Alfalfa con tres Dosis de Fertilización Fosfatada (factor B)}

\subsubsection{Etapa de Emergencia}

En la tabla 5 se indica que el porcentaje de germinación, longitud de raíz y días a la germinación no presentaron diferencias significativas $(\mathrm{P}>0.05)$ en los tres niveles de fertilización fosfatada y en el tratamiento control $(0,50,100$ y $150 \mathrm{~kg} / \mathrm{P})$ en las variedades de alfalfas introducidas.

Tabla 4. Comportamiento agronómico de la alfalfa bajo el efecto de tres niveles de fertilización fosfatada en la etapa de emergencia

\begin{tabular}{lcccccccccc} 
& \multicolumn{1}{c}{ Niveles de Fósforo } & & \\
\cline { 2 - 7 } \multicolumn{1}{c}{ Variables } & 0 & 50 & 100 & 150 & & E.E. & Prob. \\
\hline Germinación (\%) & 94.78 & a & 94.44 & a & 95.00 & a & 93.67 & a & 0.58 & 0.41 \\
Longitud raíz 30 días (cm.) & 4.67 & a & 4.67 & a & 5.56 & a & 5.67 & a & 0.37 & 0.12 \\
Días germinación & 19.44 & a & 19.67 & a & 20.56 & a & 20.11 & a & 0.27 & 0.14 \\
\hline
\end{tabular}

Letras iguales no difieren significativamente según Tukey $(\mathrm{P}<0.05)$.

Esto se debe a la la calidad física y genética de las semillas de las variedades de alfalfa fueron similares ya que existe una estrecha asociación entre la eficiencia de la implantación y genética de los alfalfares.

\section{Etapa de desarrollo}

En la tabla 6 se indica que el número de días para la formación de los tres foliolos, altura macollos 40, 60 y 90 días, número macollo 60 y 90 días de edad, estas variables no presentan diferencias significativas ( $>$ > 0.05) para los niveles del fósforo en las variedades de alfalfa, esto se debe a que las condiciones climáticas y edáficas fueron homogéneas.

Tabla 5. Comportamiento agronómico de la alfalfa bajo el efecto de tres niveles de fertilización fosfatada en la etapa de desarrollo

Variables

Niveles de Fósforo

E.E. Prob. 
Vol. 3, N³, p. 178-194, julio - septiembre, 2019

\begin{tabular}{lccccccccccc}
\hline & 0 & 50 & & 100 & & 150 & & \\
\hline Días tres foliolos & 25.00 & a & 24.56 & a & 25.56 & a & 25.11 & a & 0.49 & 0.56 \\
Días primer macollo & 33.44 & a & 33.44 & a & 33.44 & a & 33.22 & a & 0.38 & 0.97 \\
Altura macollos 40 días (cm.) & 12.14 & a & 12.40 & a & 12.26 & a & 12.26 & a & 0.33 & 0.96 \\
Altura macollos 60 días (cm.) & 23.50 & a & 23.57 & a & 23.17 & a & 24.00 & a & 0.44 & 0.62 \\
Número macollos 60 días (cm.) & 3.44 & a & 3.11 & a & 2.89 & a & 3.56 & a & 0.29 & 0.38 \\
Número macollos 90 días & 6.81 & a & 8.43 & a & 8.36 & a & 8.06 & a & 0.49 & 0.11 \\
Altura planta 90 días (cm.) & 46.51 & a & 45.18 & a & 42.83 & a & 46.62 & a & 1.36 & 0.21 \\
\hline
\end{tabular}

Letras iguales no difieren significativamente según Tukey $(\mathrm{P}<0.05)$.

\section{Etapa cosecha (110 días)}

En la tabla 7 , se indica que la altura de la planta, número de plantas $\mathrm{m}^{2}$, número y altura de rebrotes $(\mathrm{cm})$, composición botánica $(\%)$, longitud raíz $(\mathrm{cm})$ y beneficio/ costo, en la cual se observó que no existen diferencias significativas ( $p>0.05$ ) para los niveles de fertilización fosfatada $(0,50,100$ y $150 \mathrm{~kg} / \mathrm{P})$.

\section{Cobertura aérea $(\%)$}

La utilización de $150 \mathrm{~kg} / \mathrm{P} / \mathrm{ha}$ registró una cobertura aérea de $84,29 \%$, valor que difirió significativamente $(\mathrm{P}<0.01)$ de los diferentes niveles de fertilización fosfatada, puesto que al utilizar 100,50 y $0 \mathrm{~kg} / \mathrm{P} / \mathrm{ha}$, registraron valores de $82.17,78.04$ y $76.60 \%$ respectivamente.

Debiendo manifestarse que, a medida que se incrementó los niveles de fertilización fosfatada, la cobertura aérea tuvo un comportamiento directamente proporcional, de esta manera se observó una tendencia de primer orden, en donde por cada nivel de fósforo aplicado al cultivo de alfalfa, la cobertura aérea se incrementó en $0.054 \%$. Lo que permite mencionar que la disponibilidad de fósforo $(\mathrm{P})$ es uno de los factores determinantes del resultado productivo de las pasturas de alfalfa, afectando particularmente el crecimiento Christian, (1977); (Jones, 1993) la calidad (a través de la concentración de fósforo en planta (Petit, et al. 1992) y la capacidad de fijación de nitrógeno (Racca, et al. 2001). Lemache, (2015) al aplicar té de estiércol en la producción de alfalfa reportó de $94.26 \%$, valores superiores a la presente investigación, eso probablemente se deba a que el abono en base de fósforo es lenta hasta alcanzar las vellosidades de las raíces; Aragadvay, (2010) al evaluar la cobertura aérea de la alfalfa empleando niveles de bacterias Rhizobium meliloti y de estiércol de cuy; más vinaza, registraron respuestas entre 33.83 y $38.79 \%$; en el primer corte y de 24.50 a $29.37 \%$, en el otro, valores inferiores a los de la presente investigación. 
Vol. 3, N³, p. 178-194, julio - septiembre, 2019

Tabla 6. Comportamiento agronómico de la alfalfa bajo el efecto de tres niveles de fertilización fosfatada en la etapa de cosecha

\begin{tabular}{lcccccccccc}
\hline & \multicolumn{10}{c}{ Niveles de Fósforo } \\
\cline { 2 - 9 } \multicolumn{1}{c}{ Variables } & 0 & 50 & 100 & 150 & & E.E. & Prob. \\
\hline Altura planta (cm) & 53.99 & a & 54.19 & a & 52.28 & a & 55.63 & a & 1.35 & 0.40 \\
Número plantas m $^{2}$ & 17.78 & a & 18.44 & a & 18.67 & a & 21.78 & a & 1.03 & 0.06 \\
Número rebrotes & 4.89 & a & 4.89 & a & 5.33 & a & 4.44 & a & 0.27 & 0.18 \\
Altura de rebrotes (cm) & 6.11 & a & 6.78 & a & 7.11 & a & 7.00 & a & 0.38 & 0.29 \\
Cobertura aérea (\%) & 76.60 & d & 78.04 & c & 82.17 & b & 84.29 & a & 1.36 & 0.00 \\
Cobertura basal (\%) & 25.74 & d & 27.42 & c & 27.64 & b & 29.41 & a & 0.68 & 0.01 \\
Composición botánica (\%) & 94.02 & a & 93.71 & a & 95.44 & a & 94.44 & a & 0.64 & 0.28 \\
Relación hoja/tallo & 1.11 & c & 1.18 & b & 1.18 & b & 1.26 & a & 0.02 & 0.00 \\
Producción forraje verde (t/ha) & 8.00 & a & 9.46 & a & 10.04 & a & 10.25 & a & 0.77 & 0.20 \\
Producción materia seca (t/ha) & 2.02 & a & 2.46 & a & 2.28 & a & 2.58 & a & 0.18 & 0.18 \\
Longitud raíz (cm) & 16.89 & a & 17.67 & a & 19.00 & a & 18,78 & a & 0,85 & 0.89 \\
Beneficio / Costo (\$) & 1.23 & b & 1.32 & a & 1.35 & a & 1.38 & a & 0.01 & 0.02 \\
\hline
\end{tabular}

Letras iguales no difieren significativamente según Tukey $(\mathrm{P}<0.05)$.

\section{Cobertura basal (\%)}

Cuando se aplicó $150 \mathrm{~kg} / \mathrm{P} / \mathrm{ha}$ se alcanzó una cobertura basal de $29,41 \%$, valor que difirió significativamente $(\mathrm{P}<0.05)$ de los niveles de fertilización fosfatada 100,50 y $0 \mathrm{~kg} / \mathrm{P} / \mathrm{ha}$, puesto que registraron valores de $27.64,27.42$ y $25.74 \%$ respectivamente; de esta manera se puede indicar que a medida que se incrementó los niveles de fertilización fosfatada, la cobertura basal se incrementó proporcional, determinándose una tendencia lineal, en donde por cada nivel de fósforo aplicado al cultivo de alfalfa, la cobertura basal se incrementó en 0,022\%.

Lo que permite mencionar que el fósforo es imprescindible en el cultivo de alfalfa, de esta manera se corrobora a lo señalado por (Gutiérrez, 2007) quien indica que la fertilización fosfórica es muy importante en el periodo de establecimiento del cultivo, de esta manera se asegura el desarrollo radicular y el incremento de la cobertura basal, debido a que el fósforo se desplaza lentamente en el suelo, por lo que se recomienda aplicar en el momento de la siembra.

\section{Relación hojas/tallo a los 110 días de edad}

La relación hojas/tallo cuando se utilizó $150 \mathrm{~kg} / \mathrm{P} /$ ha reportó una relación de 1.26 hojas/tallo, valor que difirió significativamente $(\mathrm{P}<0.01)$ del resto de tratamientos, puesto que al utilizar 100,50 y $0 \mathrm{~kg} / \mathrm{P} / \mathrm{ha}$, alcanzó valores de $1.18,1.18$ y 1.11 hojas/tallo respectivamente, debiendo señalar que a medida que se incrementó los niveles de fertilización fosfatada, la relación 
hojas/tallo tiene un comportamiento lineal, en donde por cada nivel de fósforo aplicado al cultivo de alfalfa, la relación hojas tallo se incrementó en 0.001, ver figura 1.

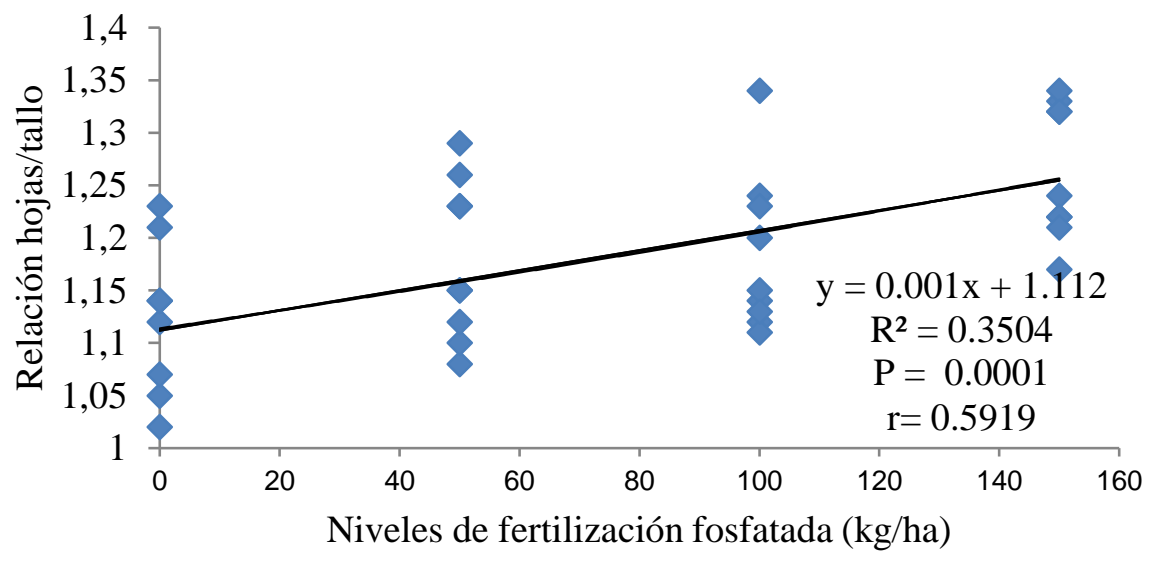

Figura 1. Relación hojas/tallo de la alfalfa a los 110 días de edad.

Romero (2002) observó que a medida que avanzó el estado de madurez del cultivo, hay un aumento en la producción ( $\mathrm{kg} / \mathrm{ms} / \mathrm{ha}$.), mientras que la calidad de la alfalfa representada por la relación hojas/tallo disminuyo como consecuencia de una menor proporción de hojas con respecto a los tallos. También se indican diferencias en producción y para la relación hoja/tallo entre épocas climáticas.

\subsubsection{Producción forraje verde y materia seca (t/ha.)}

La producción de forraje verde y materia seca en los tres niveles de fertilización fosfatada y en el tratamiento control $(0,50,100$ y $150 \mathrm{~kg} / \mathrm{P})$ no registraron diferencias significativas $(\mathrm{P}>0.05)$ reportándose producciones de 8 a 10 (t/ha/fv/corte) y de 2,02 a 2,58 (t/ha/ms $/$ corte) lo que indicaría que el manejo agronómico fue homogéneo. Horrocks y Vallentine, (1999) mencionan que la capacidad que posee una pradera para producir materia seca (MS), dependen de la disponibilidad de nutrientes, agua y, principalmente, del grado de intercepción de la radiación solar por las hojas. Con el aumento en la cantidad de hojas, se tiene una mayor intercepción de luz, pero las hojas en los estratos inferiores reciben menor intensidad y calidad de luz, por lo que provocan la reducción del crecimiento o de la tasa de asimilación neta; por ello, el mayor rendimiento de los forrajes, coincide con el mayor índice de área foliar y la mayor masa foliar verde (Morales et al., 2006).

\subsubsection{Beneficio/costo $(\$)$}

Cuando se utilizó 50, 100 y $150 \mathrm{~kg} / \mathrm{P} / \mathrm{ha}$ se obtuvo un benéfico/costo de $1.32,1.35$ y 1.38 dólares respectivamente, valores que difirieron significativamente $(\mathrm{P}<0,05)$ del tratamiento control, puesto que al no utilizar fósforo se obtuvo un beneficio/costo de 1.23 dólares; por lo que se puede indicar que a medida que se incrementó los niveles de fertilización fosfatada, el beneficio 
costo tuvo un comportamiento lineal en donde por cada nivel de fósforo aplicado el beneficio/costo fue incrementándose. Por otra parte, es importante reflexionar sobre la magnitud del beneficio económico que se determinó en cada uno de los niveles de fertilización fosfatada, respecto a la ventaja que podría representar una alternativa de invertir en la producción de forraje de alfalfa en el cantón Riobamba.

\section{CONCLUSIONES}

- La duración de las tres etapas fenológicas en el primer corte fue de 110 días en las tres variedades de alfalfas (abunda verde, cuf101 y sw8210), con una duración de 21 días en la etapa inicial y 89 días en la etapa de desarrollo. No se observó la etapa del preboton floral, por lo tanto no existió floración en los cultivos y la época de cosecha se determinó por el número y altura de los nuevos rebrotes.

- La variedad que mejor comportamiento agronómico alcanzó es la abunda verde puesto que registró una relación hojas/tallo de 1.23, lo que garantiza mayor calidad de forraje, puesto que en las hojas se dispone de mayor aporte de proteína y digestibilidad de la materia seca. La utilización de $150 \mathrm{~kg}$ de fertilización fosfatada permitió registrar una cobertura aérea de $84.29 \%$, la cobertura basal fue de $29.41 \%$, con una relación hojas/tallo de 1.26 y un beneficio/costo de $\$ 1.38$.

- La época de cosecha de las variedades de alfalfa se hizo cuando aparecen los nuevos rebrotes ya que estas especies no presentaron inducción a la floración, por lo tanto los estudios deben basarse en el tiempo que aparecen los nuevos rebrotes ya que la edad al corte influye de manera directa sobre el comportamiento vegetativo y reproductivo de la alfalfa.

\section{Referencias Bibliográficas}

Agrocalidad. (2016). Ministerio de Agricultura y Ganadería del Ecuador. Laboratorio de Análisis de suelos y fertilizantes. Análisis químico del suelo de Tunshi.

Alaska. (2015). Semillas de pastos de clima frío [Internet], [02 de marzo 2018]. http://www.imporalaska.com/26- semillas_de_pastos_de_frio.html.

Aragadvay, R. (2010) . Efecto de la aplicación de diferentes niveles de bacterias Rhizobium meliloti con la adición de estiércol de cuy en la producción forrajera del Medicago sativa. Tesis de grado. Riobamba.

Avci, M.; Ozkose, A. y Tamkoc, A. (2013). Determination of yield and quality characteristics of alfalfa (Medicago sativa L.) varieties grown in different locations. J. Na. Vet. Adv. 12(4):487-490.

Banco Central del Ecuador (2014). Estadísticas de comercio exterior. [Internet], [15 diciembre 2018]. Disponible en: http://www.portal.bce.fin.ec/vto_bueno/comercio exterior.jsp, 2014. 
Christian, K. (1977). Effects of the environment on the growth of alfalfa. Advances in Agronomy.

Ensiye; A.; Jamshid, R. y Morteza, Z. (2018). Effect of salt stress on the growth and accumulation of alfalfa ions (Medicago sativa L.). Journal of Plant Nutrition, 41: 7, 818831, DOI: 10.1080 / 01904167.2018.1426017.

Fan,J.; Wang, Q.; Malhi, S. y Li, E . (2014). Effect of mulch on the storage of water in the soil and its depletion by alfalfa in the Loess Plateau in northwestern China. Agricultural Journal Manage water. 138 (2014), pp. 10 - 6.

Grijalva, J. (1995). Producción y utilización de pastizales en la región interandina del Ecuador. Quito, Ecuador. INIAP. p. 1-10;35.

Guillén, S; Terrazas, T; De la Barrera, E y CASAS, A. (2010). Germination differentiation patterns of wild and domesticated columnar cacti in a gradient of artificial selection intensity. México. Genet Resour Crop Evol. Research Article.

Gutiérrez, I. (2007). Cultivo de alfalfa. [Internet], [10 de junio 2018] http://riegoenalfalfa.blogspot.com. Santiago de Chile.

Hawkins, C Y Long, X. (2018). Recent progress in alfalfa (Medicago sativa L.) genomics and genomic selection. United States Department of Agriculture-Agricultural Research Service, Plant Germplasm Introduction and Testing Research, Prosser, WA 99350, USA. The Crop Journal 6 (2018) 565- 575.

Harris, W. (1978). Defoliation as determinat of the growth, persistense and conposition of pasture. Australia: CSIRO.

Horrocks, R. y Vallentine, J. (1999). Harvested Forages. Academic Press. Oval Road. London. United Status of America. 426 p.

Hoyos, P.; Gracia, O. y Torres, M. (1996). Capacitación tecnológica de producción de pastos. Manejo y utilización de pasturas en Colombia. CIAT.

Infoagro. (2005). Manejo y Cultivo de la Alfalfa. [Internet], [13 de abril 2018] Disponible en: http://www.infoagro.com/herbaceas/forrajes/Alfalfa2.asp. Consultado 6 febrero 2016.

Inec. (2009). Instituto Nacional de Estadística y Censos. Encuesta de superficie y producción agropecuaria en la provincia de Chimborazo. Ministerio de Agricultura zona III.

Inamhi. (2017). Estación Meteorológica Facultad de Recursos Naturales, ESPOCH. Riobamba. Infostat. (2017). Software estadístico InfoStat versión 2017, FCA, Universidad Nacional de Córdoba. [Internet] En: www.infostat.com.ar, consulta: 10 octubre 2017.

Liu, D.; Liu, G y Yang, Z. (2015). The effect of sowing and harvesting factors on the yield of hay and the proportion of stem leaves of Medicago sativa. Acta Prataculturae Sinica. 24: $48-57$.

León, R. (2003). Pastos y Forrajes, producción y Manejo. Editorial Científica. Quito- Ecuador.

Jagush, K.; Gumbrell, M.; Mobley y Jay, N. (1976). Effect on growth of early weaning lambs onto pure lucerne stand. N.Z. J. Exp. Agric. 5:19-22.

Jones, S. (1993). Stand dynamics and yield components of alfalfa as affected by phosphorus fertility. Agronomy Journal. 
Lemache, P. (2015). Utilización de diferentes té de estiércol en la producción de Medicago sativa (alfalfa), variedad flor morada. Riobamba.

Martins, D.; Macovei, A.; Leonetti, P.; Balestrazzi, A. y Araújo, S. (2017). La influencia de fosfato, deficiencia en leguminosas. symbiotic $\mathrm{N}_{2}$ fijación. En: Sulieman S., Tran LS. (eds) DOI: https://doi.org/10.1007/978-3-319-55729-83.

Marijana,T.; Svetislav, P., Sonja, G.; Tihomir, C. y Snjezana, B. (2011). Implementation of Molecular Markers Diversity in Parental Selection of Alfalfa (Medicago sativa L.) Germplasm, Biotechnology \& Biotechnological Equipment, 25:2, 2310-2314, DOI: 10.5504/BBEQ.2011.0047.

Milic, D.; Karagic, S.; Vasiljevic, A.; Mikic, B.; Milo y Katic. S. (2014). Breeding and improvement of quality traits in alfalfa (Medicago sativa L.). Genetic 46: 11-18.

Morales, A.; Jiménez, V.; Velasco, V.; Villegas, A.; Enríquez, V. y Hernández, A. (2006). Evaluación de 14 variedades de alfalfa con fertirriego en la mixteca de Oaxaca. Técnica Pecuaria en México. 44(3):277-288.

Parker, K. (1954). Application of ecology in the determination of range condition and trend. Journal of Range Management. 7: 14-23.

Petit, P.; Pesant, L.; Barnett, O. y Dionne, M. (1992). Quality and morphological characteristics of alfalfa as affected by soil moisture, $\mathrm{pH}$ and phosphorus fertilization. Can. J. Plant Science.

Racca,L.; Collino, T; Dardanelli, O; Basigalup, L.; González, P. y Brenzoni, H. (2001). Contribución de la fijación biológica de nitrógeno a la nutrición nitrogenada de la alfalfa en la región pampeana. INTA. Argentina.

Raza, A.; Moghadda, M.; Gollner, G y Jfriedel, F. (2014). Evaluation of Crop Syst for studying the effect of mulching with Lucerne (Medicago sativa L.) in Austria, Journal of Plant Interactions, 9:1, 592-598, DOI: 10.1080/17429145.2013.877601.

Rojas, G.; Torres, S.; Joaquín, C.; Hernández, G.; Maldonado, P. \& Sánchez, P. (2017). Componentes del rendimiento en variedades de alfalfa. Agrociencia 51: 697-708.

Romero, N. (2002). $25^{\circ}$ Congreso Argentino de Producción Animal, Bs. As. [Internet], [16 de septiembre 2018] http://www.produccion-animal.com.ar. INTA Rafaela.

Ruiz, I.; G. Chahin, y C. Pedraza. (1994). Variación de la composición química y digestibilidad de algunos forrajes durante su temporada de uso en dos lecherías de la Región Metropolitana. Agricultura Técnica Chile.

Toledo, J. (1982). Manual para la evaluación agronómica: Red internacional de evaluación de pastos tropicales. Centro Internacional de Agricultura Tropical (CIAT), Cali, CO. 155 p. $\begin{array}{lllll}\text { Serie CIAT } & \text { 07sG-1 } & \text { (82) } & \text { [Internet] }\end{array}$ library.ciat.cgiar.org/articulos_ciat/Manual_Evaluacion.pdf.

Sanz, S.; Morales, F.; Arrese, I. y Aranjuelo, I. (2017). Phosphorus deficiency: An important limiting factor for rhizobial symbiosis. In: Sulieman S., Tran LS. (eds). DOI: https://doi.org/10.1007/978-3-319-55729-82.

Sas Institute. (2001). SAS User's Guide. Release 8.1. 6th ed. SAS Institute, Inc. Cary, NC. USA. 
Soto, P. y Jahn, L. (1993). Use of irrigated lucerne in different growth stages. Evaluation under cutting. New Zealand, Hamilton.

\section{PARA CITAR EL ARTÍCULO INDEXADO.}

Oñate Viteri, W., \& Flores Mariazza, E. (2019). Efecto de la fertilización fosfatada en la fenología de tres variedades de alfalfas (Medicago sativa L.). Ciencia Digital, 3(3), 178-194. https://doi.org/10.33262/cienciadigital.v3i3.622

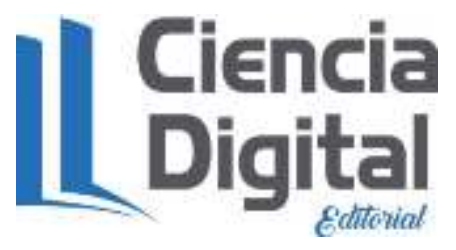

El artículo que se publica es de exclusiva responsabilidad de los autores y no necesariamente reflejan el pensamiento de la Revista Ciencia Digital.

El artículo queda en propiedad de la revista y, por tanto, su publicación parcial y/o total en otro medio tiene que ser autorizado por el director de la Revista Ciencia Digital.
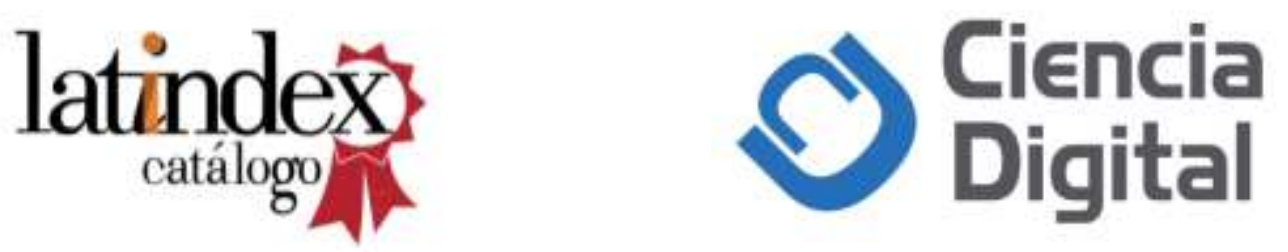